\title{
Fixed point theorems for rational type $F$-contraction
}

\begin{abstract}
Acar Ö.
In this paper, we consider rational type $F$-contraction for multivalued integral type mapping on a complete metric space. Using Wardowski's technique, we establish the existence of a fixed point of the multivalued integral type mapping, if this mapping or the $F$-contraction is continuous. In the end, we give an example which shows that our result is the best.

Key words and phrases: fixed point, multivalued mapping, F-contraction of rational type, complete metric space.
\end{abstract}

Selçuk University, Konya, Turkey

E-mail: acarozlemeymail.com

\section{Introduction}

The beginning of metrical fixed point theory is related to the Banach contraction principle, presented in 1922 [4], which says that any contraction selfmap of $M$ has a unique fixed point whenever $(M, d)$ is complete. Afterwards, the crucial role of the principle in existence and uniqueness problems arising in mathematics has been realized which fact directed the researchers to extend and generalize the principle in many ways. No doubtly, one of those ways is integral type contraction which was defined by Brianciari A. [7]. For the other techniques the reader can see the works $[8,10,18]$. In the studies of generalizations and modifications of contractions, an interesting generalization was given by Wardowski D. [19], who used a new concept of $F$-contraction. Then many authors gave some results using this concept in different types of metric spaces.

In the last decades, fixed point theory of set-valued mappings is a branch of mathematics which has received a superb attention and has different applications in differential equations, optimal control theory and convex optimization. Therefore, it lies at the crossroad of topology, theory of functions and nonlinear functional analysis. Nadler S.B. [13] was the first author, who combined the ideas of multi-valued mappings and contractions and proved that this type of mapping has a fixed point.

In this paper, we apply a rational type $F$-contraction to a multivalued integral type mapping on a complete metric space. Using Wardowski's technique, we study the existence of a fixed point of the multivalued integral type mapping if this mapping or F-contraction is continuous. 


\section{Preliminaries}

Let $(M, \mathfrak{d})$ be a metric space. $P(M)$ represent the family of all nonempty subsets of $M$, $C B(M)$ represent the family of all nonempty, closed and bounded subsets of $M$ and $K(M)$ represent the family of all nonempty compact subsets of $M$. Obviously, $K(M) \subseteq C B(M) \subseteq$ $P(M)$.

For $A, B \in C B(M)$, let

$$
\mathcal{H}(A, B)=\max \left\{\sup _{x \in A} D(x, B), \sup _{y \in B} D(y, A)\right\},
$$

where $D(x, B)=\inf \{\mathfrak{d}(x, y): y \in B\}$. Then $\mathcal{H}$ is a metric on $C B(M)$, which is called PompeiuHausdorff metric induced by $\mathfrak{d}$. We can find detailed information about Pompeiu-Hausdorff metric in $[2,6]$.

Using $\mathcal{H}$-metric, Nadler S.B. [13] present the notion of setvalued contraction mapping and proved that on complete metric space, setvalued mapping has a fixed point. Then researchers gave some results on this direction [5,9,12,15-17]. In this paper, we use Wardowski's technique, which was given in [19].

Let $(0, \infty)=\mathbb{R}^{+} . F: \mathbb{R}^{+} \rightarrow \mathbb{R}$ belongs to $\mathcal{F}$ if:

$(F 1) F$ is strictly increasing;

(F2) for each sequence $\left\{t_{n}\right\}$ of positive numbers,

$$
\lim _{n \rightarrow \infty} t_{n}=0 \text { if and only if } \lim _{n \rightarrow \infty} F\left(t_{n}\right)=-\infty ;
$$

(F3) there exists $\kappa \in(0,1)$ such that $\lim _{\alpha \rightarrow 0^{+}} \alpha^{\kappa} F(\alpha)=0$;

(F4) $F(\inf A)=\inf F(A)$ for all $A \subset(0, \infty)$ with inf $A>0$.

Definition 1 ([19]). Let $(M, \mathfrak{d})$ be a metric space and $f: M \rightarrow M$ be a mapping. Then $f$ is an $F$-contraction if $F \in \mathcal{F}$ and there exists $\tau>0$ such that

$$
\mathfrak{d}(f x, f y)>0 \Rightarrow \tau+F(\mathfrak{d}(f x, f y)) \leq F(\mathfrak{d}(x, y)) \quad \forall x, y \in M
$$

Theorem 1 ([19]). Let $(M, \mathfrak{d})$ be a complete metric space and let $f: M \rightarrow M$ be an F-contraction. Then $f$ has a unique fixed point in $M$.

Then considering the $\mathcal{H}$-metric, some fixed point results are obtained in $[11,12,14,18]$.

Lemma 1. Let $\gamma, \theta \in C B(M)$, then

$$
D(x, \theta) \leq \mathcal{H}(\gamma, \theta)
$$

for any $x \in \gamma$. 


\section{Main result}

\subsection{A fixed point theorem using Hausdorff metric}

Definition 2. Let $(M, \mathfrak{d})$ be a metric space and $f: M \rightarrow K(M)$ be a mapping. Given $F \in \mathcal{F}$ we say that $f$ is a rational type $F$-contraction of generalized multivalued integral type mapping if there exists $\tau>0$ such that

$$
\mathcal{H}(f y, f z)>0 \Longrightarrow \tau+F\left(\int_{0}^{\mathcal{H}(f y, f z)} \varkappa(s) d s\right) \leq F\left(\int_{0}^{M(y, z)} \varkappa(s) d s\right)
$$

for $y, z \in M$, where

$$
M(y, z)=\max \left\{\begin{array}{l}
\mathfrak{d}(y, z), D(y, f y), D(z, f z), \\
\frac{D(y, f y) D(z, f z)}{1+\mathfrak{d}(y, z)}, \frac{D(y, f y) D(z, f z)}{1+\mathcal{H}(f y, f z)}
\end{array}\right\}
$$

and $\varkappa: \mathbb{R}^{+} \rightarrow \mathbb{R}^{+}$is a Lebesque integrable mapping, which is summable on each compact subset of $\mathbb{R}^{+}$, nonnegative and

$$
\int_{0}^{\varepsilon} \varkappa(s) d s>0
$$

for each $\varepsilon>0$.

Theorem 2. Let $(M, \mathfrak{d})$ be a complete metric space and $f: M \rightarrow K(M)$ be a rational type $F$-contraction of generalized multivalued integral type mapping. If $f$ or $F$ is continuous, then $f$ has a fixed point.

Proof. Let $y_{0} \in M$ be an arbitrary point and define a sequence $\left\{y_{n}\right\}$ by $y_{n+1} \in f y_{n}$ for $n=1,2, \ldots$. As $f y$ is nonempty for all $y \in M$, we can choose $y_{1} \in f y_{0}$. If $y_{1} \in f y_{1}$, then $y_{1}$ is a fixed point of $f$. Let $y_{1} \notin f y_{1}$, then $D\left(y_{1}, f y_{1}\right)>0$ since $f y_{1}$ is compact. By using $(F 1)$, from Lemma 1 and (1), we can write

$$
\begin{aligned}
& F\left(\int_{0}^{D\left(y_{1}, f y_{1}\right)} \varkappa(s) d s\right) \leq F\left(\int_{0}^{\mathcal{H}\left(f y_{0}, f y_{1}\right)} \varkappa(s) d s\right) \leq F\left(\int_{0}^{M\left(y_{0}, y_{1}\right)} \varkappa(s) d s\right)-\tau \\
& \quad=F\left(\int_{0}^{\max \left\{\mathfrak{o}\left(y_{0}, y_{1}\right), D\left(y_{0}, f y_{0}\right), D\left(y_{1}, f y_{1}\right), \frac{D\left(y_{0}, f y_{0}\right) D\left(y_{1}, f y_{1}\right)}{1+\mathfrak{o}\left(y_{0}, y_{1}\right)}, \frac{D\left(y_{0}, f y_{0}\right) D\left(y_{1}, f y_{1}\right)}{1+\mathcal{H}\left(f y_{0}, f y_{1}\right)}\right\}} \varkappa(s) d s\right)-\tau \\
& \leq F\left(\int_{0}^{\max \left\{\mathfrak{o}\left(y_{0}, y_{1}\right), D\left(y_{1}, f y_{1}\right), \frac{D\left(y_{0}, f y_{0}\right) D\left(y_{1}, f y_{1}\right)}{\mathfrak{d}\left(y_{0}, y_{1}\right)}, \frac{D\left(y_{0}, f y_{0}\right) D\left(y_{1}, f y_{1}\right)}{\mathcal{H}\left(f y_{0}, f y_{1}\right)}\right\}} \varkappa(s) d s\right)-\tau \\
& \leq F\left(\int_{0}^{\max \left\{\mathfrak{o}\left(y_{0}, y_{1}\right), D\left(y_{1}, f y_{1}\right), \frac{\mathfrak{o}\left(y_{0}, y_{1}\right) D\left(y_{1}, f y_{1}\right)}{\mathfrak{d}\left(y_{0}, y_{1}\right)}, \frac{D\left(y_{0}, f y_{0}\right) D\left(y_{1}, f y_{1}\right)}{D\left(y_{1}, f y_{1}\right)}\right\}} \varkappa(s) d s\right)-\tau \\
& \leq F\left(\int_{0}^{\max \left\{\mathfrak{o}\left(y_{0}, y_{1}\right), D\left(y_{1}, f y_{1}\right)\right\}} \varkappa(s) d s\right)-\tau=F\left(\int_{0}^{\mathfrak{d}\left(y_{0}, y_{1}\right)} \varkappa(s) d s\right)-\tau
\end{aligned}
$$

Also, since $f y_{1}$ is compact, we obtain that $y_{2} \in f y_{1}$ such that

$$
\mathfrak{d}\left(y_{1}, y_{2}\right)=D\left(y_{1}, f y_{1}\right)
$$

From (2), we get

$$
F\left(\int_{0}^{\mathfrak{d}\left(y_{1}, y_{2}\right)} \varkappa(s) d s\right) \leq F\left(\int_{0}^{\mathcal{H}\left(f y_{0}, f y_{1}\right)} \varkappa(s) d s\right) \leq F\left(\int_{0}^{\mathfrak{d}\left(y_{0}, y_{1}\right)} \varkappa(s) d s\right)-\tau .
$$


If we continue recursively, we have

$$
\begin{gathered}
F\left(\int_{0}^{\mathfrak{d}\left(y_{2}, y_{3}\right)} \varkappa(s) d s\right) \leq F\left(\int_{0}^{\mathfrak{d}\left(y_{1}, y_{2}\right)} \varkappa(s) d s\right)-\tau \leq F\left(\int_{0}^{\mathfrak{d}\left(y_{0}, y_{1}\right)} \varkappa(s) d s\right)-2 \tau \\
\vdots \\
F\left(\int_{0}^{\mathfrak{d}\left(y_{n}, y_{n+1}\right)} \varkappa(s) d s\right) \leq F\left(\int_{0}^{\mathfrak{d}\left(y_{n-1}, y_{n}\right)} \varkappa(s) d s\right)-\tau \leq F\left(\int_{0}^{\mathfrak{d}\left(y_{0}, y_{1}\right)} \varkappa(s) d s\right)-n \tau .
\end{gathered}
$$

So, we get a sequence $\left\{y_{n}\right\}$ in $M$ as $y_{n+1} \in f y_{n}$ for all $n \in \mathbb{N}$. If there exists $n_{0} \in \mathbb{N}$ with $y_{n_{0}} \in f y_{n_{0}}$, then $y_{n_{0}}$ is a fixed point of $f$ and then the proof is finished. Let's say that for every $n \in \mathbb{N}, y_{n} \notin f y_{n}$. Denote

$$
a_{n}=\int_{0}^{\mathfrak{d}\left(y_{n}, y_{n+1}\right)} \varkappa(s) d s \text { for } n=0,1,2, \ldots
$$

Then, $a_{n}>0$ for all $n$. Using (3) we get

$$
F\left(a_{n}\right) \leq F\left(a_{n-1}\right)-\tau \leq F\left(a_{n-2}\right)-2 \tau \leq \cdots \leq F\left(a_{0}\right)-n \tau .
$$

From (4), we have $\lim _{n \rightarrow \infty} F\left(a_{n}\right)=-\infty$. Thus, from (F2)

$$
\lim _{n \rightarrow \infty} a_{n}=0 .
$$

From $(F 3)$, there exists $\kappa \in(0,1)$ such that

$$
\lim _{n \rightarrow \infty} a_{n}^{\kappa} F\left(a_{n}\right)=0 .
$$

By (4), the following holds for all $n \in \mathbb{N}$

$$
a_{n}^{\kappa} F\left(a_{n}\right)-a_{n}^{\kappa} F\left(a_{0}\right) \leq-a_{n}^{\kappa} n \tau \leq 0 .
$$

Letting $n \rightarrow \infty$ in (5), we obtain that

$$
\lim _{n \rightarrow \infty} n a_{n}^{\kappa}=0 .
$$

From (6), there exits $n_{1} \in \mathbb{N}$ such that $n a_{n}^{\kappa} \leq 1$ for all $n \geq n_{1}$. So, we have

$$
a_{n} \leq \frac{1}{n^{1 / \kappa}}
$$

for all $n \geq n_{1}$. Now, consider $m, n \in \mathbb{N}$ such that $m>n \geq n_{1}$ and show that $\left\{y_{n}\right\}$ is a Cauchy sequence. From (7) the following

$$
\begin{aligned}
\int_{0}^{\mathfrak{d}\left(y_{n}, y_{m}\right)} \varkappa(s) d s & \leq \int_{0}^{\mathfrak{d}\left(y_{n}, y_{n+1}\right)} \varkappa(s) d s+\int_{0}^{\mathfrak{d}\left(y_{n+1}, y_{n+2}\right)} \varkappa(s) d s+\cdots+\int_{0}^{\mathfrak{d}\left(y_{m-1}, y_{m}\right)} \varkappa(s) d s \\
& =a_{n}+a_{n+1}+\cdots+a_{m-1}=\sum_{i=n}^{m-1} a_{i} \leq \sum_{i=n}^{\infty} a_{i} \leq \sum_{i=n}^{\infty} \frac{1}{i^{1 / \kappa}} .
\end{aligned}
$$

hold. By the convergence of the series $\sum_{i=1}^{\infty} i^{-1 / \kappa}$, we get

$$
\int_{0}^{\mathfrak{d}\left(y_{n}, y_{m}\right)} \varkappa(s) d s \rightarrow 0 \text { as } n \rightarrow \infty .
$$


This yields that $\left\{y_{n}\right\}$ is a Cauchy sequence in $(M, \mathfrak{d})$. Since $(M, \mathfrak{d})$ is a complete metric space, the sequence $\left\{y_{n}\right\}$ converges to some point $z \in M$, that is, $\lim _{n \rightarrow \infty} y_{n}=z$.

If $f$ is compact, we have $f y_{n} \rightarrow f z$ and

$$
D\left(y_{n}, f z\right) \leq \mathcal{H}\left(f y_{n}, f z\right)
$$

so $D(z, f z)=0$ and $z \in f z$.

If $F$ is continuous, now we claim that $z \in f z$. Assume the contrary, that is, $z \notin f z$. So, there exist an $n_{0} \in \mathbb{N}$ and a subsequence $\left\{y_{n_{k}}\right\}$ of $\left\{y_{n}\right\}$ such that $D\left(y_{n_{k}+1}, f z\right)>0$ for all $n_{k} \geq n_{0}$ (otherwise, there exists $n_{1} \in \mathbb{N}$ such that $y_{n} \in f z$ for all $n \geq n_{1}$, which implies that $z \in f z$, this is a contradiction, since $z \notin f z)$. Since $D\left(y_{n_{k}+1}, f z\right)>0$ for all $n_{k} \geq n_{0}$, then

$$
\begin{aligned}
& \tau+F\left(\int_{0}^{D\left(y_{n_{k}+1}, f z\right)} \varkappa(s) d s\right) \leq \tau+F\left(\int_{0}^{\mathcal{H}\left(f y_{n_{k}}, f z\right)} \varkappa(s) d s\right) \leq \tau+F\left(\int_{0}^{M\left(y_{n_{k}}, z\right)} \varkappa(s) d s\right)
\end{aligned}
$$

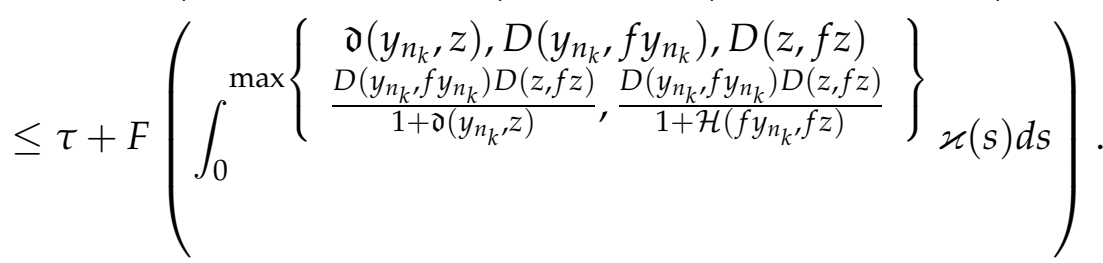

Taking the limit $k \rightarrow \infty$ and using the continuity of $F$, we have $\tau+F(D(z, f z)) \leq F(D(z, f z))$, which is a contradiction. Thus, we get $z \in \overline{f z}=f z$. This completes the proof.

Corollary 1. Let $(M, \mathfrak{d})$ be a complete metric space and $f: M \rightarrow K(M)$ be a rational type F-contraction of multivalued integral type mapping, i.e. there exists $\tau>0$ such that

$$
\mathcal{H}(f y, f z)>0 \Rightarrow \tau+F\left(\int_{0}^{\mathcal{H}(f y, f z)} \varkappa(s) d s\right) \leq F\left(\int_{0}^{\mathfrak{d}(y, z)} \varkappa(s) d s\right)
$$

for $y, z \in M$, where $\varkappa: \mathbb{R}^{+} \rightarrow \mathbb{R}^{+}$is a Lebesque integrable mapping which is summable on each compact subset of $\mathbb{R}^{+}$, nonnegative and

$$
\int_{0}^{\varepsilon} \varkappa(s) d s>0
$$

for each $\varepsilon>0$. If $f$ or $F$ is continuous, then $f$ has a fixed point.

Corollary 2. Let $(M, \mathfrak{d})$ be a complete metric space and $f: M \rightarrow K(M)$ be a rational type contraction of multivalued integral type mapping, i.e.

$$
\int_{0}^{\mathcal{H}(f y, f z)} \varkappa(s) d s \leq \int_{0}^{\mathfrak{d}(y, z)} \varkappa(s) d s
$$

for $y, z \in M$, where $\varkappa: \mathbb{R}^{+} \rightarrow \mathbb{R}^{+}$is a Lebesque integrable mapping which is summable on each compact subset of $\mathbb{R}^{+}$, nonnegative and

$$
\int_{0}^{\varepsilon} \varkappa(s) d s>0
$$

for each $\varepsilon>0$. If $f$ or $F$ is continuous, then $f$ has a fixed point. 


\subsection{A fixed point theorem using $\delta$-distance}

Let $(M, \mathfrak{d})$ be a metric space. For $\gamma, \theta \in B(M)$ we define

$$
\delta(\gamma, \theta)=\sup \{\mathfrak{d}(y, \eta): y \in \gamma, \eta \in \theta\}
$$

and

$$
D(y, \theta)=\inf \{\mathfrak{d}(y, \eta): \eta \in \theta\} .
$$

If $\gamma=\{y\}$ we write $\delta(\gamma, \theta)=\delta(y, \theta)$ and also if $\theta=\{\eta\}$, then $\delta(y, \theta)=\mathfrak{d}(y, \eta)$. It is easy to prove that for $\gamma, \theta, \varsigma \in B(M)$

$$
\begin{aligned}
& \delta(\gamma, \theta)=\delta(\theta, \gamma) \geq 0 \\
& \delta(\gamma, \theta) \leq \delta(\gamma, \varsigma)+\delta(\varsigma, \theta) \\
& \delta(\gamma, \gamma)=\sup \{\mathfrak{d}(y, \eta): y, \eta \in \gamma\}, \\
& \delta(\gamma, \gamma)=0 \text { implies that } \gamma=\theta=\{y\}
\end{aligned}
$$

If $\left\{\gamma_{n}\right\} \subset B(M)$ and $\gamma_{n} \rightarrow \gamma, \gamma \subseteq M$ if and only if

- $y \in \gamma$ implies that $y_{n} \rightarrow y$ for some sequence $\left\{y_{n}\right\}$ with $y_{n} \in \gamma_{n}$ for $n \in \mathbb{N}$ and

- for any $\varepsilon>0$, there exists $n_{0} \in \mathbb{N}$ such that $\gamma_{n} \subseteq \gamma_{\varepsilon}$ for $n>n_{0}$, where

$$
\gamma_{\varepsilon}=\{x \in M: \mathfrak{d}(x, y)<\varepsilon \text { for some } y \in \gamma\} \text {. }
$$

Lemma 2 ([3]). Let $\left\{\gamma_{n}\right\},\left\{\theta_{n}\right\} \subset B(M)$ and $(M, \mathfrak{d})$ is a complete metric space. If $\gamma_{n} \rightarrow \gamma \in$ $B(M)$ and $\theta_{n} \rightarrow \theta \in B(M)$ then $\delta\left(\gamma_{n}, \theta_{n}\right) \rightarrow \delta(\gamma, \theta)$.

Lemma 3 ([3]). If $\left\{\gamma_{n}\right\} \subset B(M)$ and $(M, \mathfrak{d})$ is a complete metric space, $\delta\left(A_{n}, y\right) \rightarrow 0$ for some $y \in M$, then $\gamma_{n} \rightarrow\{y\}$.

Also, in [1] they gave a fixed point theorem for generalized multivalued $F$-contraction mappings with $\delta$-distance as follows.

Definition 3. Let $(M, \mathfrak{d})$ be a metric space and $f: M \rightarrow B(M)$ be a mapping. Then $f$ is a generalized multivalued $F$-contraction if $F \in \mathcal{F}$ and there exists $\tau>0$ such that

$$
\tau+F(\delta(f y, f z)) \leq F(M(y, z))
$$

for all $y, z \in M$ with $\min \{\delta(f y, f z), \mathfrak{d}(y, z)\}>0$, where

$$
M_{\delta}(y, z)=\max \left\{\mathfrak{d}(y, z), D(y, f y), D(z, f z), \frac{1}{2}[D(y, f z)+D(z, f y)]\right\} .
$$

Theorem 3 ([1]). Let $(M, \mathfrak{d})$ be a complete metric space and $f: M \rightarrow B(M)$ be a multivalued $F$-contraction. If $F$ is continuous and $f y$ is closed for all $y \in M$, then $f$ has a fixed point in $M$.

Definition 4. Let $(M, \mathfrak{d})$ be a metric space and $f: M \rightarrow K(M)$ be a mapping. Then $f$ is said to be a rational type $F$-contraction of generalized multivalued integral type mapping with $\delta$-distance if $F \in \mathcal{F}$ and there exists $\tau>0$ such that

$$
\tau+F\left(\int_{0}^{\delta(f y, f z)} \varkappa(s) d s\right) \leq F\left(\int_{0}^{M_{\delta}(y, z)} \varkappa(s) d s\right)
$$


for all $y, z \in M$ with $\min \{\delta(f y, f z), \mathfrak{d}(y, z)\}>0$, where

$$
M_{\delta}(y, z)=\max \left\{\begin{array}{l}
\mathfrak{d}(y, z), D(y, f y), D(z, f z), \\
\frac{D(y, f y) D(z, f z)}{1+\mathfrak{d}(y, z)}, \frac{D(y, f y) D(z, f z)}{1+f f i(f y, f z)}
\end{array}\right\},
$$

and $\varkappa: \mathbb{R}^{+} \rightarrow \mathbb{R}^{+}$is a Lebesque integrable mapping which is summable on each compact subset of $\mathbb{R}^{+}$, nonnegative and

$$
\int_{0}^{\varepsilon} \varkappa(s) d s>0
$$

for each $\varepsilon>0$.

The following theorem can be proved in much the same way as Theorem 2 .

Theorem 4. Let $(M, \mathfrak{d})$ be a complete metric space and $f: M \rightarrow K(M)$ be a rational type $F$-contraction of generalized multivalued integral type mapping with $\delta$-distance. If $f$ or $F$ is continuous, then $f$ has a fixed point.

Corollary 3. Let $(M, \mathfrak{d})$ be a complete metric space and $f: M \rightarrow K(M)$ be a multivalued contraction if $F \in \mathcal{F}$ and there exists $\tau>0$ such that

$$
\tau+F(\delta(f y, f z)) \leq F\left(M_{\delta}(y, z)\right)
$$

for all $y, z \in M$ with $\min \{\delta(f y, f z), \mathfrak{d}(y, z)\}>0$. If $f$ or $F$ is continuous, then $f$ has a fixed point.

Corollary 4. Let $(M, \mathfrak{d})$ be a complete metric space and $f: M \rightarrow B(M)$ be a multivalued contraction if $F \in \mathcal{F}$ and there exists $\tau>0$ such that

$$
\tau+F(\delta(f y, f z)) \leq F\left(M_{\delta}(y, z)\right)
$$

for all $y, z \in M$ with $\min \{\delta(f y, f z), \mathfrak{d}(y, z)\}>0$. If $F$ is continuous and $f y$ is closed for all $y \in M$, then $f$ has a fixed point.

Example 1. Let $M=\{0,1,2,3, \ldots\}$ and

$$
\mathfrak{d}(y, z)= \begin{cases}0, & y=0 \\ y+z, & y \neq z\end{cases}
$$

Then $(M, \mathfrak{d})$ is a complete metric space. Define $f: M \rightarrow K(M)$ by the

$$
f y= \begin{cases}\{0\}, & y=0 \\ \{0,1,2,3, \ldots, y-1\}, & y \neq 0 .\end{cases}
$$

We claim that $f$ is rational type $F$-contraction of generalized multivalued integral type mapping with $\delta$-distance with respect to $F(\alpha)=\alpha+\ln \alpha, \tau=1$. Because of the

$$
\min \{\delta(f y, f z), \mathfrak{d}(y, z)\}=0,
$$

we can consider the following cases while $y \neq z$ and $\{y, z\} \cap\{0,1\}$ is empty or singleton. 
Case 1. If $y>1$ and $z=0$, then $D(0, f y)=0$ and

$$
\begin{aligned}
\tau+F\left(\int_{0}^{\delta(f y, 0)} \varkappa(s) d s\right) & =\tau+F\left(\int_{0}^{y-1} \varkappa(s) d s\right) \\
& \leq \tau+F\left(\int_{0}^{\mathfrak{d}(y, 0)} \varkappa(s) d s\right) \leq F\left(\int_{0}^{M(y, 0)} \varkappa(s) d s\right) .
\end{aligned}
$$

Case 2. If $y>1$ and $z=1$, then $D(1, f y)=0$ and

$$
\begin{aligned}
\tau+F\left(\int_{0}^{\delta(f y, f)} \varkappa(s) d s\right) & =\tau+F\left(\int_{0}^{y-1} \varkappa(s) d s\right) \leq \tau+F\left(\int_{0}^{y+1} \varkappa(s) d s\right) \\
& \leq \tau+F\left(\int_{0}^{\mathfrak{d}(y, 1)} \varkappa(s) d s\right) \leq F\left(\int_{0}^{M(y, 1)} \varkappa(s) d s\right) .
\end{aligned}
$$

Case 3. If $y>z>1$, then

$$
\begin{aligned}
\tau+F\left(\int_{0}^{\delta(f y, f z)} \varkappa(s) d s\right) & =\tau+F\left(\int_{0}^{y-1} \varkappa(s) d s\right) \\
& \leq \tau+F\left(\int_{0}^{\mathfrak{d}(y, z)} \varkappa(s) d s\right) \leq F\left(\int_{0}^{M(y, z)} \varkappa(s) d s\right) .
\end{aligned}
$$

This shows that $f$ is rational type F-contraction of generalized multivalued integral type mapping with $\delta$-distance, therefore, all conditions of Theorem 4 are satisfied and so $f$ has a fixed point in $M$.

\section{References}

[1] Acar Ö., Altun I. A fixed point theorem for multivalued mappings with $\delta$-distance. Abstr. Appl. Anal. 2014, article ID 497092. doi:10.1155/2014/497092

[2] Agarwal R.P., O'Regan D., Sahu D.R. Fixed Point Theory for Lipschitzian-type Mappings with Applications, Springer-Verlag, New York, 2009.

[3] Altun I. Fixed point theorems for generalized $\varphi$-weak contractive multivalued maps on metric and ordered metric spaces. Arab. J. Sci. Eng. 2011, 36 (8), 1471-1483. doi:10.1007/s13369-011-0135-8

[4] Banach S. Sur les opérations dans les ensembles abstraits et leur application aux équations intégrales. Fund. Math. 1922, 3 (1), 133-181.

[5] Berinde V. On the approximation of fixed points of weak contractive mappings. Carpathian J. Math. 2003, 19 (1), 7-22.

[6] Berinde V., Pacurar M. The role of the Pompeiu-Hausdorff metric in fixed point theory. Creat. Math. Inform. 2013, $22(2), 143-150$.

[7] Branciari A. A fixed point theorem formappings satisfying a general contractive condition of integral type. Int. J. Math. Math. Sci. 2002, 29 (9), 531-536, article ID 641824. doi:10.1155/S0161171202007524

[8] Ćirić Lj.B. A generalization of Banach's contraction principle. Proc. Amer. Math. Soc. 1974, 45 (2), $267-273$. doi:10.2307/2040075

[9] Ćirić Lj.B. Multi-valued nonlinear contraction mappings. Nonlinear Anal. 2009, 71 (7-8) 2716-2723. doi: 10.1016/j.na.2009.01.116

[10] Hardy G.E., Rogers T.D. A generalization of a fixed point theorem of Reich. Canad. Math. Bull. 1973,16 (2), 201-206. doi:10.4153/CMB-1973-036-0 
[11] Klim D., Wardowski D. Fixed point theorems for set-valued contractions in complete metric spaces. J. Math. Anal. Appl. 2007, 334 (1), 132-139. doi:10.1016/j.jmaa.2006.12.012

[12] Mizoguchi N., Takahashi W. Fixed point theorems for multivalued mappings on complete metric spaces. J. Math. Anal. Appl. 1989, 141 (1), 177-188. doi:10.1016/0022-247X(89)90214-X

[13] Nadler S.B. Multi-valued contraction mappings. Pacific J. Math. 1969, 30 (2), 475-488.

[14] Acar Ö., Durmaz G., Minak G. Generalized multivalued F-contractions on complete metric spaces. Bull. Iranian Math. Soc. 2014, 40 (6), 1469-1478.

[15] Reich S. Fixed points of contractive functions. Boll. Unione Mat. Ital. 1972, 5, $26-42$.

[16] Reich S. Some fixed point problems. Atti Accad. Naz. Lincei Rend. Lincei Mat. Appl. 1974, 57 (3-4), $194-198$.

[17] Suzuki T. A generalized Banach contraction principle that characterizes metric completeness. Proc. Amer. Math. Soc. 2008, 136 (5), 1861-1869. doi:10.1090/S0002-9939-07-09055-7

[18] Suzuki T. Mizoguchi-Takahashi's fixed point theorem is a real generalization of Nadler's. J. Math. Anal. Appl. 2008, 340 (1), 752-755. doi:10.1016/j.jmaa.2007.08.022

[19] Wardowski D. Fixed points of a new type of contractive mappings in complete metric spaces Fixed Point Theory Appl. 2012, article number 94 (2012). doi:10.1186/1687-1812-2012-94

Received 16.07.2020

Revised 30.01.2021

Акар О. Теореми про фіксовану тоику для F-стиску раціонального типу // Карпатські матем. публ. - 2021. - Т.13, №1. - С. 39-47.

У цій статті розглянуто F-стиск раціонального типу для багатозначного відображення інтегрального типу на повному метричному просторі. За допомогою техніки Вардовського встановлено існування нерухомої точки такого відображення інтегрального типу у випадку, коли це відображення або F-стиск раціонального типу $є$ неперервними. Також наведено приклад, який ілюструє, що основний результат є найкращим.

Ключові слова і фрази: нерухома точка, багатозначне відображення, F-стиск раціонального типу, повний метричний простір. 